\title{
Müller Glial Cells of the Goldfish Retina are Phagocytic In Vitro but not In Vivo
}

\author{
ELISABETH C. WAGNER AND PAMELA A. RAYMOND* \\ Department of Anatomy and Cell Biology, The University of Michigan, Ann Arbor. MI 48109-0616. \\ U.S.A.
}

(Received 13 September 1990 and accepted in revised form 10 January 1991)

\begin{abstract}
The role of Müller glial cells in the process of degeneration and regeneration of the goldfish retina is poorly understood. One potential role is phagocytosis of neuronal debris in degenerating retinas. We investigated the phagocytic capacity of Müller glial cells of the goldfish retina both in vitro and in vivo. Müller glial cells from primary or first passage cultures were incubated with latex beads to assess their phagocytic ability, and acridine orange staining was used to identify phagolysosomes in living Müller glial cells. These experiments showed that Müller glial cells are phagocytic in culture. Cell identity was verified with an antibody raised against glial fibrillary acidic protein (GFAP). For the in vivo experiments fluorescent latex beads alone or in combination with the metabolic poison ouabain were injected into the pusterior chamber. At various intervals ( 4 days to 8 weeks) after injection the retinas were prepared for immunocytochemistry. Polyclonal anti-GFAP and NN-1, a monoclonal antibody which recognizes macrophages and microglia within the goldfish retina, were used to identify the phagocytic cells. When the beads were injected into the eye, they were phagocytosed by macrophages/microglia cells but not by Müller cells.

Key words: goldfish retina: Müller glial cells; phagocytosis; latex beads; cell culture; retina degeneration : ouabain: immunocytochemistry.
\end{abstract}

\section{Introduction}

Phagocytic activity by Müller glial cells in vivo has been reported for various pathological conditions of the retina. Upon injection of red blood cells (Miller et al., 1986) or carbon particles (Algvere and Kock, 1983 ) into the posterior chamber of rabbit eyes, the endfeet of the Müller glial cells form protrusions and phagocytose the offending particles. In addition. phagocytosis of latex beads by human Müller glial cells in culture has been demonstrated by Mano and Puro (1990). To our knowledge, the only report of phagocytic activity in the teleost retina following injection of latex beads is by Li et al. (1987) who demonstrated phagocytosis of latex beads by pigment epithelium cells of the goldfish eye. The beads were injected into the eye after optic nerve transsection or removal of the optic tectum. No phagocytosis by Müller cells was reported.

We were interested to know whether the Müller glial cells of the goldfish retina would participate in phagocytosis of neuronal debris during experimental degeneration of the retina. Ouabain, a neurotoxin that inhibits $\mathrm{Na}^{+}, \mathrm{K}^{+}$ATPase, when injected into the posterior chamber of the goldfish eye causes degeneration of the retinal neurons (Maier and Wolburg, 1979). Retinal cells surviving the treatment include the mitotic cells of the circumferential germinal zone and the rod precursor cells, which normally give rise to rods throughout the life of the goldfish (Johns and

* For correspondence.
Fernald, 1981; Raymond et al., 1988), as well as microglia and Müller glial cells. To learn about the role of the Müller glia during degeneration we injectcd into the posterior chamber of goldfish eyes a combination of ouabain, to cause the retina to degenerate, together with fluorescent latex beads as a marker for phagocytic cells. We also injected latex beads into normal (nondegenerating) eyes to determine whether the Müller glia cells are phagocytic in a normal retina. In a third experiment, eyes were injected sequentially first with ouabain alone to cause the retina to degenerate and to stimulate phagocytic activity, followed by an injection of fluorescent latex beads 8 days later. In none of the in vivo experiments did we observe Müller glial cells containing beads.

From these results we conclude that Müller glial cells of the goldfish retina are not phagocytic for carboxylate latex beads in vivo. On the other hand, cultured Müller glia from normal and from degenerating goldfish retinas proved to be capable of phagocytosis as soon as they had migrated out of the retinal explants. All procedures were carried out in accord with the ARVO Resolution on Use of Animals in Research.

\section{Materials and Methods}

Animals

Goldfish were purchased from Ozark Fisheries (Richland, MO) and were maintained in the laboratory at room temperature. The fish selected for the in vivo 


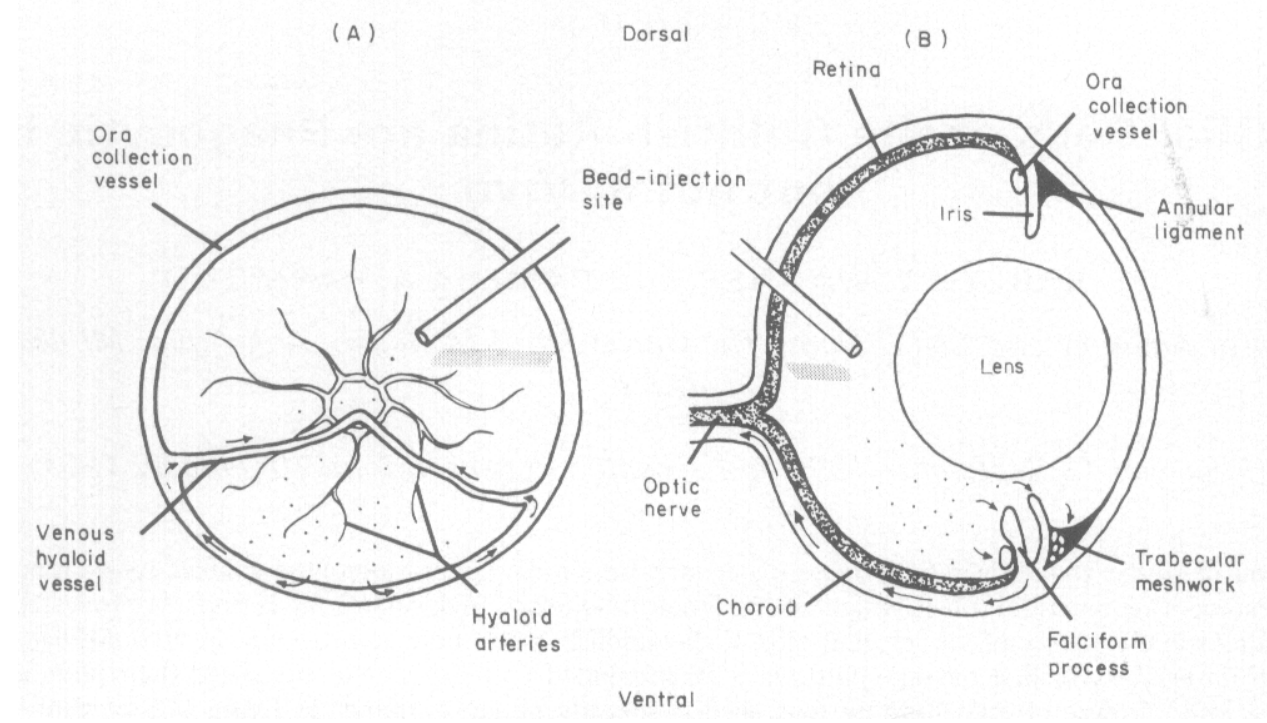

Fig. 1. A, The aqueous outlet pathways in the teleost retina are different from those in mammals. Frontal view of the retinal surface. viewed through the pupil. The major veins on the retinal surface include the ora collection vessel which empties into two venous hyaloid vessels located in the ventral half of the eye. These hyaloid vessels converge at the optic disk where they join to form the ophthalmic vein which exits the eye in association with the optic nerve. B, Radial section through the eye. The trabecular meshwork in the iris angle is similarly restricted to the ventral one-third of the circumference of the eye. The dorsal two-thirds of the iris angle is composed of the annular ligament. The pathway of aqueous outflow is directed ventrally towards the trabecular meshwork, and thence into the choroidal vessels, eventually passing into the ophthalmic vein.

experiments were $3-4 \mathrm{~cm}$ in body length. For the in vitro experiments larger fish were used $(8-10 \mathrm{~cm})$. The age of the fish was unknown but probably ranged from 1 year or less to 2 or 3 years (Johns and Easter. 1977).

\section{In Vivo Experiments}

Fish were anesthetized in $0 \cdot 2 \%$ tricaine methanesulfonate (MS 222, Sigma). A small incision was made through the sclera at the limbus on the nasal side of the eye. A Hamilton microliter syringe (33-gauge fixed needle with a blunt tip) was filled with the injection solution and attached to a micromanipulator. The right eye was injected with a mixture of ouabain (Sigma), $0.2 \mathrm{~mm}$ in $0.9 \%$ saline, and carboxylate latex beads (Fluoresbrite $\phi=0.74 \mathrm{~mm}$, conjugated to FITC; Polysciences, Warrington, PA); the left eye was injected with beads only. The final intraocular concentration of ouabain was estimated to be $10 \mathrm{~mm}$ (Raymond et al.. 1988), and the approximate number of beads injected into each eye was $5.7 \times 10^{6}$. At various times after injection ( 4 days and 2, 4 and 8 weeks), the fish were deeply anesthetized and killed by decapitation.

In a second experiment, the right eye was injected with ouabain alone $(10 \mathrm{~mm}$ intraocular concentration). Eight days later the same eye was injected with fluorescent latex beads $\left(5.7 \times 10^{6}\right.$ beads per eye). Eight days after bead injection, the fish were deeply anesthetized and killed.

Following death, both eyes were enucleated and the cornea and lens removed. The eyes were fixed in $4 \%$ paraformaldehyde with $5 \%$ sucrose in phosphate buffer $(\mathrm{pH} 7 \cdot 4)$ for $2 \mathrm{hr}$. Fixation was followed by several rinses in phosphate buffer with sucrose $(\mathrm{pH}$ $7 \cdot 4$ ). Before cryoprotection the sclera was removed, the eyes bisected and infiltrated in a graded series of sucrose $(10,15$ and $20 \%)$ in phosphate buffer and finally in a 2:1 mixture of $20 \%$ sucrose and OCT compound (Miles Laboratories, Elkhart, IN) according to Barthel and Raymond (1990). Finally, the half eyes were submerged in a $2: 1$ sucrose-OCT mixture in aluminum foil molds and then frozen in 2-methylbutane (iso-pentane) cooled in liquid nitrogen. Cryosections $3 \mu \mathrm{m}$ thick were cut on a Reichert-Jung 2800 Frigocut cryostat. The sections were placed on gelatine-coated slides, air dried and stored at $-90^{\circ} \mathrm{C}$ until they were processed for immunocytochemistry.

Immunocytochemistry. All sections were doublelabeled with antibodies selective for Müller glia and for microglia/macrophages. Non-specific binding of antibodies was first blocked with $20 \%$ normal goat serum (NGS) in phosphate buffered saline (PBS), pH 7.4. supplemented with $0.5 \%$ Triton $\mathrm{X}-100$ and $0.1 \%$ sodium azide for $20 \mathrm{~min}$. The sections were then incubated with antibodies to glial fibrillary acidic protein (bovine GFAP polyclonal, DAKO, Carpinteria, $\mathrm{CA}$ ) diluted $1: 200$ in $1 \% \mathrm{NGS}$ in PBS, pH 7.4, at $4{ }^{\circ} \mathrm{C}$ overnight. [This antibody recognizes intermediate filaments in Müller glia cells (Bignami and Dahl, 1979).] Incubation with the primary antibody was followed by two rinses in PBS with $1 \%$ NGS and $0.5 \%$ Triton X-100 for $10 \mathrm{~min}$ each. To visualize the bound antibody the sections were incubated with a rhodamine (TRITC)-conjugated anti-rabbit IgG antibody (Jackson ImmunoResearch Laboratories, West Grove. PA), preabsorbed with mouse serum proteins, at $1: 20$ dilution for $1 \mathrm{hr}$, and then the sections were washed as 
above. A short fixation in $1 \%$ paraformaldehyde in PBS (10 $\mathrm{min}$ ) before the second labeling considerably reduced non-specific binding of the rhodamineconjugated secondary antibody. After this step the excess fixative was carefully rinsed off in PBS. The sections were again incubated with $20 \%$ NGS, then labeled with the second primary antibody, NN-1 $(1: 200)$, a mouse monoclonal antibody generated against goldfish retinal homogenate and which labels microglia and macrophages in the goldfish retina (Raymond and Barthel, 1989). In addition, NN-1 also labels the pigmented layers of the iris, the retinal pigment epithelium and blood vessels. The secondary antibody used to visualize the $\mathrm{NN}-1$ labeled cells was anti-mouse $\operatorname{IgG}$ conjugated to the blue fluorochrome 7-amino-4-methylcoumarin-3-acetic acid (AMCA) and preabsorbed against rabbit serum proteins (Jackson ImmunoResearch), plated at 1:20 dilution for $1 \mathrm{hr}$, then washed. The sections were photographed on a Leitz Aristoplan fluorescent microscope.

\section{In Vitro Experiments}

Culture method. In order to produce cultures that were free of retinal pigment epithelium, the fish were dark adapted for at least $1 \mathrm{hr}$ prior to dissection of the retina. The dark-adapted retinas of large goldfish $(8-10 \mathrm{~cm}$ body length) were isolated, and care was taken to remove any adhering pigment epithelium cells. The freshly isolated retinas were stored in Dulbecco's modified PBS until all the eyes were dissected. Each retina was placed on a sterile Teflon plate and cut on a tissue chopper (Sorvall TC-2 Tissue Sectioner) into squares of 225 or $500 \mathrm{~mm}$. The retinal explants were collected in a Petri dish and rinsed in fresh Dulbecco's PBS. After the explants had settled in the dish the PBS was drawn off and replaced with M199 culture medium (Gibco Laboratories, Grand Island, NY) containing $4.1 \mathrm{~mm}$ sodium bicarbonate and $10 \%$ fetal bovine serum (FBS). The retinal explants were either immediately plated onto poly-Llysine $(0 \cdot 1 \%)$ coated-culture dishes or left floating in the medium for at least a week (Burke and Foster, 1984) before plating. Cultures were kept in air at $26^{\circ} \mathrm{C}$. Müller glia started to migrate away from the explant onto the culture dish as early as $24 \mathrm{hr}$ after attachment of the explants.

Phagocytosis experiment. Some cultures were incubated with $5 \mathrm{~mm}$ ouabain in serum-free M199 culture medium for 3-4 hr then rinsed in Hank's balanced salt solution (IIBSS) and stored in M199 culture medium with $4.1 \mathrm{~mm}$ sodium bicarbonate and $10 \%$ FBS for $24 \mathrm{hr}$ prior to the phagocytosis experiment. Primary or first passage normal and ouabain treated cultures were incubated with a $0.05 \%$ solution of polystyrene latex beads $(1.1 \mathrm{~mm}$. Sigma Chemical, St Louis, MO), diluted in M199 culture medium with $4 \cdot 1 \mathrm{mM}$ sodium bicarbonate and 10\% FBS. The cultures were incubated for 5-24 hr. The cells were washed in HBSS and then stained for phagolysosomes with the basic dye acridine orange, $0.01 \%$ in HBSS for 3 min (Zeitz. et al.. 1979). The cells were rinsed in HBSS for 3 min and immediately viewed with a Leitz Fluorovert inverted fluorescence microscope. The bead-containing phagolysosomes appear as brilliant orange fluorescent granules surrounded by pale green fluorescent cytoplasm. Since non-fluorescent beads were used for this experiment, only the internalized beads were visualized with this method. Ouabain treated cultures were incubated with fluorescent latex beads.

Immunocytochemistry. Cell cultures were either fixed in cold acetone $\left(-20^{\circ} \mathrm{C}\right)$ for $10 \mathrm{~min}$ or in $4 \%$ paraformaldehyde for $30 \mathrm{~min}$. The antibodies NN-1 and GFAP (both diluted at 1:200) were used to characterize the cell types in the cultures. The staining procedure was performed using the avidin-biotinperoxidase complex (Vectastain anti-rabbit for GFAP and anti-mouse for NN-1, Vector Laboratories. Burlingame, $\mathrm{CA}$ ).

Incubation of isolated retina. Dark-adapted retinas were isolated as above and incubated for 4-24 hr in culture medium containing Fluoresbrite carboxylate latex beads $\left(1.75 \mathrm{~mm}, 2.8 \times 10^{6}\right.$ beads $\left.\mathrm{ml}^{-1}\right)$. Another group of retinas was first incubated in serum-free M199 tissue culture medium containing 10 or $50 \mathrm{~mm}$ ouabain for $3 \mathrm{hr}$, then stored in M199 with $10 \%$ FBS for $24 \mathrm{hr}$. After this, the retinas were incubated in M199 tissue culture medium containing beads at the same concentration as above. During all these steps the retinas were gently agitated on an orbital shaker. The retinas were rinsed in PBS and then fixed in $4 \%$ paraformaldehyde for $1 \mathrm{hr}$ and processed for frozen sections or for light microscopy.

\section{Results}

\section{In Vivo Experiments}

For all the in vivo experiments at least five fish were examined at each time point.

Normal eyes injected with latex beads. By direct visualization through the pupil, we determined that the injected fluorescent latex beads initially distributed evenly in the posterior chamber of the eye. At the time of dissection, however, the beads had accumulated in the ventral half of the eye. Some had entered the anterior chamber and were concentrated in the area of the trabecular meshwork [Figs 1 (A) and (B)]. [The trabecular meshwork is limited to the ventral onethird of the circumference of the eye in the goldfish (Tripathi, 1974: Raymond et al., 1984.] Radial sections of eyes 4 days after bead-injection showed massive accumulations of beads in the ventral half of 


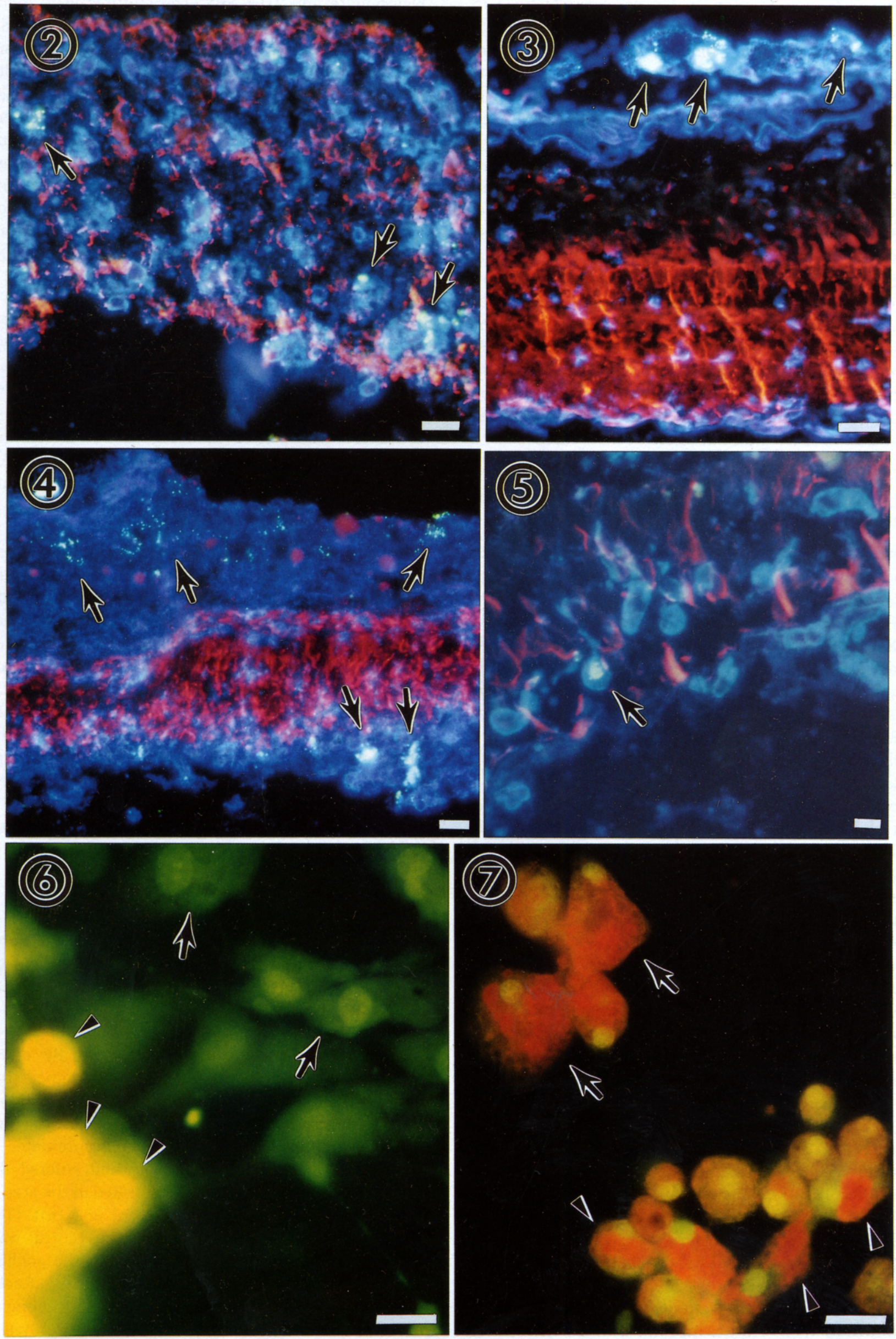


the eye adjacent to the ora collection vessel as well as in the ventral part of the iris and in the most ventral part of the choroid. Some beads were located in the venous hyaloid vessels and in the choroid (Fig. 3). Many beads were still free in the posterior chamber and some had been incorporated by macrophages in the vitreous. Very few $\mathrm{NN}-1$ reactive cells at the vitreal surface of the retina contained beads. No beads could be observed in the GFAP stained Müller glia cells or in microglia within the layers of the retina.

At 2 weeks after injection fewer beads were in the vitreous but a larger section of the ventral choroid was filled with beads. In other respects the result was the same as at 4 days. Four weeks after injection some beads were localized within the ophthalmic vein. At 4 and 8 weeks after injection, beads were still trapped in the iris, the choroid and the trabecular meshwork. At none of the time points examined could beads be localized in GFAP stained cells or within microglia in the retina. From these observations we conclude that the Müller glia cells in the normal cye do not phagocytose latex beads.

Simultaneous injection of ouabain and beads. In retinas 4 days after a combined injection of ouabain and beads. some beads were phagocytosed either by microglia or by macrophages (Fig. 2). The neural layers of the retina are destroyed at this time point, and since the antibody (NN-1) recognizes both microglia resident in the retina and wandering macrophages, we cannot distinguish between these two cells types in the degenerated retina. The $\mathrm{NN}-1$ positive phagocytic cells were localized within the mass of degenerating retina. No beads were found in GFAP positive Müller glia cells. which at this point had retracted their radial processes (Fig. 2). Two weeks after injection the retina had already begun to regenerate and the Müller glia had formed short radial processes, spanning the width of the regenerating retina (Raymond et al., 1988). Rare bead-containing NN-1 reactive cells were found in the subretinal space or at the vitreal surface of the retina. At none of the examined times $\lfloor 4$ days, 2 weeks (Fig. 4), 4 weeks and 8 weeks] were beads observed inside GFAP positive cells.
Degeneration with ouabain followed by bead injection. One could argue that the above test for phagocytic activity by Müller glia cells gave a negative result because after simultaneous injection of the neurotoxin ouabain and the latex beads, the beads might be phagocytosed by macrophages in the vitreous or filtered into non-retinal tissue before the Müller glia were stimulated to phagocytose particles. For this reason we next destroyed the neural retina 8 days before injecting beads. However, even with cellular debris already present by the time the beads were injected, we did not observe phagocytic activity of Müller glia (Fig. 5). Compared to the distribution of beads in eyes at 2 weeks after simultaneous ouabain and bead injections, there were considerably more beads within macrophages in the vitreous and degenerated retina and fewer in the iris and choroid in the sequentially injected eyes. This demonstrates that macrophages had proliferated upon retinal degeneration and/or the aqueous outflow dynamics of the eyc were disrupted by the ouabain treatment.

\section{In Vitro Experiments}

Isolated whole retinas. Neither in the isolated normal retinas nor in the retinas that had been treated with ouabain prior to incubation with fluorescence latex bead were phagocytic Müller glia cells observed.

Explant cultures. As soon as the retinal explants adhered to the substrate of the culture dish, neurites grew out and large epithelioid cells migrated out of the explant. The large epithelioid cells were classified as Müller glia by their reaction with an antibody against bovine GFAP, an intermediate filament protein (Fig. 8). In the goldfish retina this antibody reacts only with Müller glial cells (Raymond and Barthel, 1989). In first passage cultures more than $90 \%$ of the migrated cells reacted with GFAP. Other cells with a morphology different from the GFAP reactive cells were NN-1 positive. The $\mathrm{NN}-1$ positive cells were smaller and more rounded than the GFAP positive cells, and they had numerous short spiny processes, whereas Müller glia had very long processes. The NN-1 positive cells

FIG. 2. Radial section through the retina 4 days after simultaneous injection of beads and ouabain. Note the beads in NN1 positive (blue) cells (arrows). GFAP positive Müller glia are red. Bar $=20 \mu \mathrm{m}$.

FIG. 3. Radial section through the retina 4 days after bead injection. Beads (arrows) have been phagocytosed by NN-1 positive (blue) cells in the choroid. GFAP positive Müller glia are red. Bar $=20 \mu \mathrm{m}$.

FIG. 4. Radial section through the retina 2 weeks after simultaneous injection of ouabain and beads. Beads (arrows) have either been phagocytosed by NN-1 positive (blue) macrophages in the posterior chamber or have entered the NN-1 positive blood vessels of the choroid. No beads were found within the layers of the regenerating retina or within GFAP positive cells. Bar $=20 \mu \mathrm{m}$.

FIG. 5. Radial section through an eye injected sequentially with ouabain and then injected with beads. Note the beads (arrow) in the NN-1 positive cell. Bar $=20 \mu \mathrm{m}$.

FIg. 6. Acridine orange stain in living explant culture. The macrophages (arrowheads) in the explant are bright yelloworange. Müller cells (arrows) have migrated out; they show pale green background fluorescence. Bar $=20 \mu \mathrm{m}$.

FIG. 7. Acridine orange stained living culture $4 \mathrm{hr}$ after adding latex beads. Large, flat migratory Müller cells (arrows) and smaller, round macrophages (arrowheads) have incorporated latex beads as indicated by the bright orange staining of phagolysosomes. Bar $=20 \mu \mathrm{m}$. 


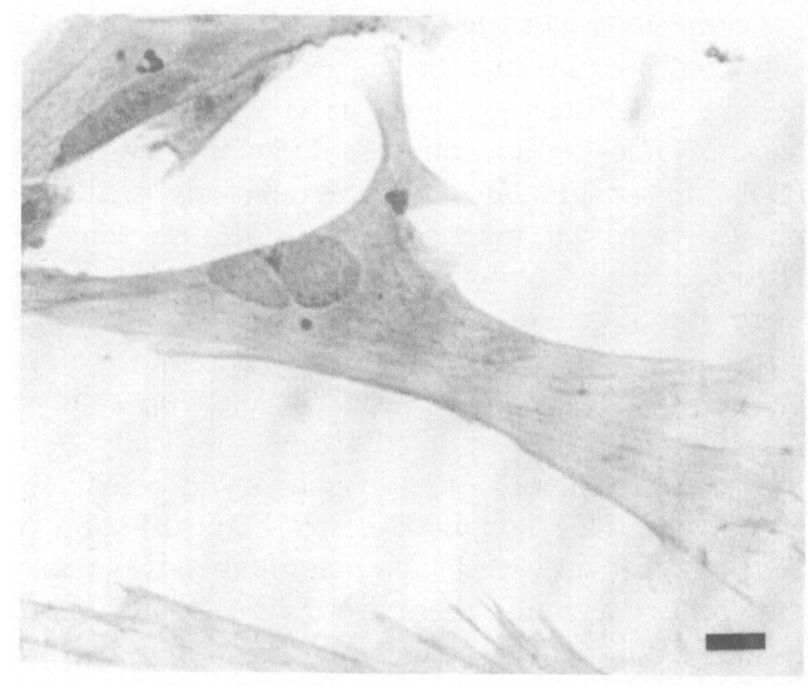

FiG. 8. GFAP antibody, visualized with biotinylated peroxidase, confirms that these large, flat migratory cells are Müller cells. Bar $=20 \mu \mathrm{m}$.

were often sitting on top of a migratory Müller cell or were very close to the explant.

In living cultures vitally stained with acridine orange, a large number of cells at the edges or in the nearest neighborhood of the explant contained large phagolysosomes which stained bright yellow-orange (Fig. 6). These cells were identified as microglia and/or macrophages on the basis of their morphology. The Müller glia cells, which were located at greater distances from the explant and had a characteristic flattened shape, contained only a few, very small orange spots around the nucleus. In contrast, when the primary cultures were exposed to non-fluorescent latex beads for 5-24 hr, the Müller glia cells contained many large phagolysosomes stained with acridine orange (Fig. 7). This result demonstrated that both normal and ouabain-treated cultured Müller glia were capable of phagocytosing latex beads soon after they had become migratory.

\section{Discussion}

These results show that Müller glia cells of the goldfish retina are phagocytic for latex beads when they are isolated in cell cultures but not when they are in contact with other cells within the retina. We do not know whether the altered phenotypic expression is a result of the changes in cell shape, the different molecular environment or the degree of contact with other cells.

The biological significance of phagocytosis by Müller glia in the normal retina is questionable. Transportation of phagocytosed particles through the layers of the retina and release of the heads into the subretinal space is an inefficient way to remove particles from the posterior chamber of the eye, although this pathway has been described in rabbit retina (Burke and Foster, 1982). These authors observed transretinal migration by vitreal macrophages marked with latex beads. Some senescent macrophages lysed within the layers of the retina and the free spheres were then phagocytosed by outer portions of the Müller glia cells.

In the goldfish eye two pathways for removal of cellular debris and injected latex beads were observed. First, the aqueous outflow system from the posterior chamber into the anterior chamber offers an efficient way to remove beads from the vitreous. The conventional outflow pathway from the anterior chamber consists of the trabecular meshwork in the ventral one third of the iris angle and the intra- and episcleral plexus of collector channels including the aqueous veins (Tripathi, 1974: Raymond et al., 1984). Accessory drainage routes include the uveo-scleral drainage and diffusion across iris vessels (Tripathi. 1974). A second pathway for removal of debris is via macrophages of the vitreous, which phagocytose the beads and enter the veins on the retinal surface. The engorged macrophages leave the posterior chamber through the ora collection vessel, the venous hyaloid vessel and the ophthalmic vein.

The injected beads did not cause pathological changes in the goldfish eye, such as expansion of the Müller glia and formation of protrusions into the posterior chamber. Neither proliferation nor formation of an epiretinal membrane was observed in goldfish as has been described following injection of ink (Algvere and Kock, 1983) or blood cells (Miller et al., 1986) into rabbit eyes.

When injection of ouabain was followed by injection of beads. not as many beads were found in the iris. the choroid and the trabecular meshwork as compared to simultaneous injection of beads and toxin. One reason might be that ouabain treatment not only affects neurons but also the circulation system in the posterior chamber and the blood vessels. Another reason might be that in sequentially injected eyes the degenerating retina, and thus the accumulation of neuronal debris. induces proliferation of macrophages in the vitreous. Therefore, at the time of bead injection there may have been abundant macrophages present to phagocytose the beads before they could flow into the anterior chamber.

At 4 days after simultaneous injection of beads and ouabain we observed some phagocytic cells within the mass of the degenerating retina. We do not know whether the NN-1 positive cells were resident microglia or wandering macrophages since our monoclonal antibody NN-1 reacted with both cell types. There are two principal forms of microglia in the CNS, the ameboid or reactive microglia which is morphologically and functionally similar to vascular monocytes or macrophages (Rio-Hortega, 1932: Ling, 1981: Oehmichen, 1983) and the ramified microglia which appears during late postnatal period in the CNS and is not capable of engulfing particles (Oehmichen. 1983). It has been suggested that the ameboid cells differentiate into ramified cells during post-natal 
development (Ling, 1981 ; Giulian and Baker, 1986). The location and shape of resting or ramified microglia within the adult rabbit retina have been demonstrated with enzyme-histochemical methods for thiamine pyrophosphatase (TPPase) and nucleoside diphosphatase (NDPase) (Schnitzer, 1989). In goldfish retina, the NN-1 reactive cells cannot be demonstrated with enzyme bistochemistry, although they are located in the same layers of the retina as ramified microglia of other vertebrates (Raymond and Barthel, unpub. obs.). Therefore, the NN-1 reactive cells within the normal goldfish retina may be ameboid microglia that have never differentiated into ramified microglia. Changing patterns of distribution of $\mathrm{NN}-1$ reactive cells have been observed following experimentally induced degeneration of the goldfish retina (Raymond and Barthel. 1989). This could be another indication that the microglia of the goldfish retina remain ameboid and retain their phagocytic ability. The fact that the distribution of the microglia is not altered after bead injection in a normal eye might be an indication that the injected latex beads never penetrate into the retina.

Ameboid microglia isolated from rat brain begin to develop processes after a few days in culture. At the same time they gradually reduce their phagocytic activity (Giulian and Baker, 1986). This suggests that ameboid microglia can differentiate into ramified microglia in culture. With the formation of processes and their transformation into ramified microglia the cells lose the enzymatic activity of non-specific esterases. The reason for the observation that Müller glia cells are phagocytic when migratory and without radial processes, but are not phagocytic when integrated in the retina, might be based on a similar relationship between structure, enzymatic activity, and phagocytic ability.

Based on our results, the question of whether the Müller glia cells of the goldfish retina are phagocytic or not has to be answered both yes and no. The different results obtained from cell culture experiments and from injections into the eye demonstrate that experiminents in a culture system cannot always replace in vivo studies.

\section{Acknowledgements}

This work was supported by NIH grants R01-EY04318 (to P.A.R.) and T32-EY07022 (E.C.W.). Pamela A. Raymond has published previously as P.R. Johns. We thank Linda Barthel and Tanya Gibson for expert technical assistance.

\section{References}

Algvere, P. and Kock, E. (1983). Experimental epiretinal membranes induced by intravitreal carbon particles. Am. J. Ophthalmol. 102, 188-195.
Barthel, L. K. and Raymond, P. A. (1991). Improved method for obtaining $3 \mu \mathrm{m}$ cryosections for immunocytochemistry. J. Histochem. Cytochem. (in press).

Bignami, A. and Dahl, D. (1979). The radial glia of Müller in the rat retina and their response to injury. $\Lambda \mathrm{n}$ immunofluorescence study with antibody to the glial fibrillary acidic (GFA) protein. Exp. Eye Res. 28, 63-9.

Burke, J. M. and Foster, S. J. (1982). Ultrastructural demonstration of transretinal migration by vitreal macrophages marked with latex particles. Ophthalmic Res. 14. $317-25$.

Burke. J. M. and Foster. S. J. (1984). Culture of adult rabbit retinal glial cells: methods and cellular origin of explant outgrowth. Curr. Eye Res. 3, 1169-77.

Giulian, D. and Baker, T. J. (1986). Characterization of ameboid microglia isolated from developing mammalian brain. Neuroscience 6, 2163-78.

Johns. P. R. and Easter, S. S. (1977). Growth of the adult goldfish eye. II. Increase in retinal cell number. J. Comp. Neurol. 176, 331-42.

Johns, P. R. and Fernald, R. D. (1981). Genesis of rods in teleost fish. Nature 293, 141-2.

Li, W. W. Y., Hui, B. S. W., Tsang, D. S. C. and Yew, D. T. (1987). Optic nerve transsection and tectal removal affect phagocytic activity of the pigment epithelium in goldfish. Acta Histochem. 81, 149-53.

Ling, E. A. (1981). The origin and nature of microglia. In Advances in Cellular Neurobiology, Vol. 2. (Fedoroff, S. and Hertz, L.). Pp. 33-82. Academic Press: New York.

Maier, W. and Wolburg, H. (1979). Regeneration of the goldfish retina after exposure to different doses of ouabain. Cell Tiss. Res. 202, 99-108.

Mano, T. and Puro, D. G. (1990). Phagocytosis by human retinal glial cells in culture. Invest. Ophthalmol. Vis. Sci. $31,1047-55$.

Miller, B., Miller, H. and Ryan, S. J. (1986). Experimental epiretinal proliferation induced by intravitreal red blood cells. Am. J. Ophthalmol. 102, 188-95.

Oehmichen, M. (1983). Inflammatory response in the central nervous system. Prog. Neuropathol. 5, 277-325.

Raymond, P. A. and Barthel, L. K. (1989). Retinal regeneration in goldfish: use of cell specific monoclonal antibodies to assess the reestablishment of normal cytoarchitecture. Soc. Neurosci. Abst. 15, 808.

Raymond, P. A., Reifler, M. J. and Rivlin, P. K. (1988). Regeneration of goldfish retina: rod precursors are a likely source of regenerated cells. J. Neurobiol. 19. 431-63.

Raymond, P. A., Spilman, R., Hill, R. and Bahn, C. (1984). The telescopic eyes of Black Moor goldfish: elevated intraocular pressure and altered aqueous outflow pathways. Invest. Ophthalmol. Vis. Sci. 25, (Suppl.), 282.

Rio-Hortega, P. del, (1932). Microglia. In Cytology and Cellular Pathology of the Nervous System. Vol. 2. (W. Penfield, Ed.). Pp. 481-584. Paul P. Hocker: New York.

Schnitzer. J. (1989). Enzyme-histochemical demonstration of microglial cells in the adult and postnatal rabbit retina. J. Comp. Neurol. 282, 249-63.

Tripathi, R. C. (1974). Comparative physiology and anatomy of the aqueous outflow pathway. VII. Osteichthyes (Bony fish). In The Eye Vol. 5. (Dawson, $\mathrm{H}$. and Graham. L. T. Eds.). Pp 309-18. Academic Press: New York.

Zeitz, M., Lange, K., Keller, K. and Herken, H. (1979). Distribution of acridine orange accumulating particles in neuroblastoma cells during differentiation and their characterization by subcellular fractionation and electron microscopy. Cell Mol. Biol. 25, 305-25. 